\title{
Le développement au féminin : un projet de société
}

Analyses de projets de la Direction de la coopération au développement et de l'Aide humanitaire (DDA)

\section{Françoise Lieberherr-Gardiol}

\section{(2) OpenEdition}

Édition électronique

URL : http://journals.openedition.org/aspd/1347

DOI : $10.4000 /$ aspd. 1347

ISSN : 1663-9669

\section{Éditeur}

Institut de hautes études internationales et du développement

\section{Édition imprimée}

Date de publication : 1 janvier 1988

Pagination : 243-259

ISSN : 1660-5934

\section{Référence électronique}




\title{
Le développement au féminin: un projet de société
}

\author{
Analyses de projets de la Direction \\ de la coopération au développement \\ et de l'Aide humanitaire (DDA)
}

\author{
Françoise Lieberherr-Gardiol
}

"Les femmes soutiennent la moitié du cie/» Mao Tse Tung

Les femmes représentent la moitié de l'humanité et pourtant, parler des femmes se révèle un discours souvent difficile et délicat. Dans ce thème passionnant à débattre, j'aimerais éviter les voies extrêmes et sans issue que sont les dénonciations polémiques ou l'autosatisfaction bureaucratique, les accusations revendicatrices ou un humanitarisme trop naiif.

Par contre, il m'intéresse d'observer le parcours des femmes: reconnaître les points de repère, décrire l'état de la route, signaler les obstacles et les virages. En d'autres termes, tenter de répondre à des questions élémentaires: pourquoi la route des femmes dans le développement n'est-elle pas droite? Et pourquoi y at-il des limitations de vitesse entraînant des freinages et des accélérations?

\section{Un fort taux d'ambiguilté}

Quand on aborde la problématique des femmes dans le développement, on perçoit qu'elle est affectée de ce que j'appelerai un fort ataux d'ambiguïté». Il y a ambiguïté entre la femme participant à la production économique et la femme presque invisible dans les statistiques, entre le rôle de la femme proclamé théoriquement et dévalorisé concrètement dans la pratique quotidienne. Il y a ambiguilté entre les 
modèles culturels qui immobilisent les femmes par des déterminismes historiques et des pesanteurs sociales, et les aspirations nouvelles d'une société qui se transforme rapidement. II y a ambiguïté aussi parce que la société industrielle mesure al'efficacité du développement sur des facteurs aux mains des hommes, tels l'argent, les machines, les technologies avancées, et non sur des facteurs contrôlés par les femmes tels que l'éducation des enfants, l'alimentation familiale, ou la transmission du patrimoine culturel collectif. Ily a ambiguïté encore parce qu'il n'existe pas une femme moyenne au pluriel, mais qu'il existe sur la planète des femmes très différentes les unes des autres. II y a ambiguïté parce que les uns pensent accorder des privilèges et les autres pensent demander des droits.

Ainsi ce taux d'ambiguilté enferme la femme dans des stéréotypes d'infériorité qui aggravent ses conditions de travail, qui dévalorisent son existence sociale, et qui l'écartent le plus souvent des décisions et des responsabilités.

Même la terminologie utilisée dans les projets de développement traduit cette ambiguïté. Certains parlent de «prise en considération» de la femme dans le développement, ce qui renforcerait un rôle féminin relativement passif. D'autres s'expriment en termes d' $\alpha$ intégration $*$ de la femme, qui pourrait signifier une simple normalisation aux valeurs dominantes. D'autres encore le définissent comme «participation» au développement, attribuant à la femme un rôle plus actif sur la scène que dans les coulisses.

\section{Vers une féminisation de la société}

De manière générale dans la société occidentale contemporaine, le droit à la parole pour les femmes se fait jour progressivement. La déclaration d'une Décennie de la femme en 1975 a officialisé un courant d'idées en préparation depuis de nombreuses années, et a légitimé des proclamations d'égalité compatibles avec la dignité humaine (1967) (1). En outre la multiplication des études, des discours et des messages d'information a mis en évidence l'existence biologique, la complémentarité économique et la responsabilité socio-culturelle des femmes, qui «soutiennent la moitié du ciel » dans toutes les sociétés, dans toutes les catégories sociales, dans toutes les familles. Et cette féminisation de la société encore timide tend de plus en plus à abandonner les «stratégies offensives des -ismes» qui marquent notre civilisation industrielle (de l'impérialisme au colonialisme et militarisme par le féminisme militant de la première heure). Au lieu de perpétuer des rapports de force, elle s'exprime en valeurs positives de complémentarité sociale.

Et pourtant dans cette marche en avant subsistent des résistances et des difficultés: inégalités de salaire, discriminations dans la formation, surcharges de travail, pertes de responsabilités traditionnelles. Et les retombées des crises économiques de la décennie 80 aggravent leurs conditions dans une nouvelle tendance de féminisation de la pauvreté (2). Tous ces aspects de la réalité quotidienne contribuent à amplifier la marginalisation de la femme dans le développement et sa dépendance, à l'opposé des principes proclamés et définis dans les projets, c'est-à-dire intégration et participation. 
Malgré les nouveaux critères philosophiques définis dans les déclarations des organisations internationales, les différentes formes de discrimination sexuelle ne se sont pas transformées d'un coup en aégalitarisme sexuel». Pourquoi la féminisation dans le développement rencontre-t-elle tant d'obstacles, et pourquoi n'est-elle pas comprise comme une rééquilibration fondamentale des rapports humains? Pourquoi la aféminitude» dans le développement ne serait-elle pas perçue comme une dimension positive et constructive à l'équilibre des rapports sociaux? L'explication globale est simple mais les mécanismes en sont complexes: parce qu'il ne s'agit pas d'un problème de femmes mais d'un problème de société. La participation de la femme est inscrite dans un projet collectif.

C'est pourquoi pour traiter cette problématique, nous allons braquer les projecteurs non sur la catégorie femmes qui évoluerait selon une dynamique strictement féminine. Une dynamique féminine est artificielle. Nous allons éclairer le champ de la société où interagissent hommes et femmes, et mettre en lumière ses déterminismes économiques, ses mécanismes sociaux et ses codes culturels pour déceler les rouages cachés sous les pratiques sociales.

\section{A la DDA, pour des actions globales et intégrées}

Ily a plusieurs années déjà que la DDA chemine à pas mesurés vers la participation des femmes dans les pays en développement. Quelques jalons:

- Depuis plus de vingt ans, 23 projets de développement au financement de un million et plus ont intégré la composante femme à travers la santé, la nutrition, l'éducation-formation, la création de revenus et les activités villageoises, dans une perspective de développement rural intégré. Cette prise en considération explicite de la femme dans le développement en Afrique, en Amérique latine et en Asie, s'est considérablement développée depuis dix ans.

- 1982, définition des lignes directrices de la DDA: «Participation de la femme au développement w. Celles-ci soulignent d'une part les problèmes des femmes, et d'autre part leur apport fondamental au développement dans leurs responsabilités économiques et sociales au sein de la famille et de la collectivité. Malgré la diversité des normes culturelles dans le monde définissant les statuts et les situations des femmes, leurs rôles restent déterminants partout comme partenaires d'un développement pour le bénéfice de la société entière dont elles font partie. Les lignes directrices proposent comme règle principale de la coopération que la responsabilité du développement soit assumée par la société locale, par les acteurs femmes et hommes auxquels la DDA apporte conseil et appui, expériences d'analyse, de gestion et d'ouverture aux problèmes socioéconomiques. Dans le changement culturel que doit opérer la société ellemême, la participation des femmes ne peut se réaliser que si on se préoccupe de leurs besoins, si on tient compte des facteurs socio-culturels en jeu, si on donne aux femmes des capacités d'actions concrètes (3). 
- 1987, définition d'un programme sectoriel alntégration des femmes dans le développement».

- Depuis une dizaine d'années, participation active à l'intégration des femmes dans les assemblées internationales (conférence de Nairobi, politiques femmes à l'UNICEF, l'OMS, l'OCDE).

- Depuis quinze ans, sensibilisation générale auprès de tous les collaborateurs de la DDA sur les dimensions multiples des femmes dans le développement, femmes omniprésentes dans la famille, dans le village, dans le pays (4). Loin des revendications militantes, cette sensibilisation met en évidence qu'on reconnaît explicitement le rôle et la participation de la femme dans le développement. En deux principes-clés: la femme est une partenaire de la production économique et de la reproduction sociale, aux deux niveaux de la famille et de la société. La femme est un élément structurel de l'ensemble d'une société, un pôle d'une totalité bipolaire homme-femme.

- Cette évolution en marche doit se poursuivre dans l'avenir par l'élaboration de méthodes et d'instruments, qui contribuent à former les collaborateurs et à concrétiser la participation des femmes dans le développement.

En outre, ces préoccupations sur la participation de la femme correspondent aux principes fondamentaux de la "philosophie DDA», soit promouvoir un développement intégré et global qui contribue à la cohérence culturelle et à la solidité sociale des collectivités. Sa mise en œuvre passe par le soutien des catégories sociales les plus défavorisées où se situent les femmes, et par la participation active des acteurs primaires et leur responsabilisation dans le développement.

\section{Des pléges à éviter}

Mais cette prise en considération de la femme dans le développement doit éviter des pièges qu'on pourrait schématiser ainsi: ethnocentrisme, homogénéisation sociale, acculturation figée, réduction socialisante du rôle des femmes. Plus en détails, le piège de transposer directement nos modèles de développement issus de l'industrialisation et de l'urbanisation occidentale à des sociétés aux schémas agraires, aux valeurs culturelles non occidentales, aux projets collectifs peu techniques et productivistes. Autre piège, celui d'homogénéiser la réalité sociale des pays en développement sans distinguer des acteurs différenciés aux intérêts divergents et aux rôles souvent conflictuels: urbains et ruraux, intellectuels, commerçants et paysans, leaders et masses populaires. Troisième piège, celui de percevoir l'acculturation comme un fait lié à la colonisation et non comme une acculturation permanente à travers le processus du développement. L'acculturation opère en continu par le mélange des valeurs culturelles exogènes et endogènes qui définissent la dynamique sociale des pays en développement. Dernier piège, réduire la participation de la femme au développement à un rôle secondaire d'assistance sociale, béquille pour une démarche masculine, et non à son rôle socio-culturel inhérent à la marche d'une société sur deux jambes valides, l'une masculine et 
l'autre féminine. Ces quatre pièges qui guettent toute coopération au développement se résument à une question initiale et fondamentale à poser et reposer dans toutes les interventions: où et comment existent, parlent et agissent les femmes dans les pays en développement?

La prise en considération de la femme dans le développement progresse certes dans la DDA, mais elle n'est pas exempte de lenteurs et contradictions (5). Or les meilleures recommendations ne suffisent pas. C'est pourquoi je propose de comprendre la nature des obstacles et des limitations de vitesse en étudiant deux expériences telles qu'elles se sont déroulées dans le terrain, sur la scène et dans les coulisses.

\section{Au Tchad, les Centres de Formation Professionnelle Agricole (CFPA) du Moyen-Chari}

Les CFPA au Moyen-Chari dans le sud du Tchad ont été créés en 1964 pour promouvoir un développement rural intégré dans cette zone par l'introduction de la culture attelée et de nombreuses techniques liées à cette nouvelle pratique. L'action des CFPA s'est basée sur une conception de développement global et intégré qui valorise une notion de progrès qualitatif, où l'amélioration des conditions de vie ne dépend pas d'un seul changement sectoriel technique ou économique, mais résulte d'une transformation plus complète qui touche aux éléments sociaux et culturels des villageois.

Dans cette longue et intéressante expérience (6), nous ne mentionnerons que quelques points de repère concernant la participation des femmes et nous en ferons une brève analyse. La lecture en trois niveaux successifs, c'est-à-dire première lecture des faits, deuxième lecture des démarches, et troisième lecture des effets, nous permet de mettre en évidence progressivement les mécanismes sociaux et de les situer dans une dynamique de transformation de ces collectivités.

Cette expérience de plus de vingt ans qui a survécu aux graves perturbations socio-politiques des dernières années, a permis de mettre en scène les femmes tchadiennes. Une animation féminine en milieu rural tchadien dans les années 1972-73, soutenue par la DDA et assurée par des acteurs et actrices africains représente en soi une expérience ad'avant-garde» sur le terrain du développement. En outre, cette animation féminine réalisée depuis plus de dix ans, a expérimenté en quelque sorte les objectifs d'une action femmes et les conditions sociales de sa réalisation.

\section{Falts}

Création en 1964 des CFPA pour former une élite de jeunes paysans susceptibles d'entraîner à leur retour le reste de la population vers le progrès agricole.

\section{Première réorientation en 1971:}

- formation de couples paysans pour améliorer la diffusion des techniques agricoles en les intégrant dans la famille, unité de production primaire. 
- création d'une animation féminine destinée à répondre aux besoins des femmes.

$2 \theta$ réorientation en 1977 privilégiant 3 aspects:

- formation de jeunes ménages d'agriculteurs comme relais d'innovations dans leurs villages.

- promotion d'actions communautaires autocentrées choisies par les villageois.

- renforcement de la formation féminine.

3 évaluations de l'expérience CFPA ontété réalisées en 1971, 1977 et 1985, comme réflexion sur leur évolution, leur bilan et leurs projets.

En 20 ans, 2'500 familles ont été formées dans les Centres, $\infty$ qui représente au moins 10'000 personnes touchées.

100 villages ont été touchés par les actions communautaires.

4'500 personnes (soit $13 \%$ de la population active) ont été touchées par l'alphabétisation fonctionnelle en langue sara (383 alphabétisateurs).

Le fonctionnement des CFPA, dont le personnel est tchadien avec l'appui de 4 coopérants européens, comprend:

- une direction régionale assurant la gestion administrative

- des Centres de formation avec des moniteurs et monitrices assurant la formation des délégués villageois (couples et leurs enfants) et l'animation dans les villages.

L'animation féminine porte sur la santé, l'hygiène, la nutrition, la couture, la fabrication du savon local, l'alphabétisation fonctionnelle.

\section{Démarches}

L'action de formation ne s'est pas réalisée de manière linéaire, mais par ajustements successifs: changements d'objectifs et de priorités en fonction des résultats, c'està-dire tâtonnements et corrections selon le principe essai-erreur.

\section{En phases discontinues.}

action plus ou moins rapides ou plus ou moins lentes avec des temps de réalisation et de diffusion différents (par exemple, entre construction de puits, alphabétisation, stockage de semences, gestion de pharmacies villageoises, etc.).

L'action de développement par la formation est liée à des innovations.

La conception d'animation féminine correspondait à une innovation aux 2 niveaux de la Suisse et du Tchad.

- A la DDA, le principe d'animation féminine s'imposa progressivement. Ensuite l'engagement d'une animatrice pour former des monitrices, qui soit non seulement femme mais africaine, fut accepté par la DDA après discussion. Sont intervenus comme facteurs positifs la formation suisse (Fribourg) d'une Togolaise boursíère comme maîtresse d'école ménagère, et la formation suisse du responsable tchadien des CFPA favorable à cette idée. 
- Au Tchad dans le milieu rural du Moyen-Chari, les innovations furent d'instituer un «enseignement" par des monitrices agentes de développement, et de former des monitrices à diverses matières et techniques nouvelles. Innovations dans le contenu de l'animation, par exemple alphabétisation fonctionnelle, éducation sur la santé et la transmission des maladies, introduction de nouveaux modes d'alimentation basés sur les valeurs nutritives des aliments, etc.

L'innovation en tant qu'idée nouvelle est introduite de l'extérieur puis diffusée.

Par exemple l'idée de l'animation féminine fut lancée par un opérateur européen ayant déjà une expérience du Moyen-Chari.

Les actions de développement sont des réponses aux besoins des collectivités. En exemple, le besoin d'intégrer la formation agricole, destinée d'abord aux jeunes hommes célibataires, dans les ménages en stage annuel, à cause du rôle primordial de la femme dans les activités paysannes, et à cause de l'importance de la famille comme lieu traditionnel de reproduction économique et sociale.

La diffusion des innovations est assurée par des agents locaux.

Les monitrices sont originaires des villages, éduquées dans les valeurs traditionnelles, et intégrées dans la réalité locale culturelle. Elles sont acceptées comme connaissant les besoins de la population et ne menaçant pas les valeurs de la société locale.

\section{Effets}

Dans cette expérience menée sur plus de 20 ans, les femmes sont intégrées dans la formation au développement. Cette participation féminine est caractérisée par:

Un décalage. Décalage entre une formation initiale destinée aux hommes selon un schéma occidental de modernisation agricole et l'intégration de la femme dans la formation des ménages presque 10 ans après. Situation renforcée par le décalage dans la formation scolaire locale, particulièrement en milieu rural où seulement quelques filles sont scolarisées.

Une certaine marginalisation du rôle des femmes. La formation aux activités agricoles et commerciales est réservée aux hommes. Les femmes demandent une formation en agriculture et en alphabétisation pour maîtriser les outils techniques du développement et bénéficier d'un petit revenu économique monétaire (marché).

Un impact différencié. L'animation féminine a touché les femmes du Moyen-Chari de manière différenciée d'après leurs degrés de participation, selon 3 niveaux d'actrices:

- les monitrices, agentes de développement, non seulement sont très bien formées dans les techniques du développement à l'intention des femmes, mais elles sont capables de les enseigner dans les CFPA aux couples stagiaires et dans les villages (activités communautaires).

- les femmes déléguées villageoises formées dans les CFPA, qui devraient être des relais du développement rural auprès des villageoises, ne savent utiliser que 
peu les connaissances reçues et ne savent pas les transmettre aux autres villageoises. Le mécanisme de la atache d'huile" ne fonctionne pas. Un handicap majeur réside dans la logique du choix des délégués par les villageois qui désignent des hommes. Les femmes accompagnent leur mari et se révèlent souvent peu motivées et trop timides pour animer (problème additionnel des couples monogames admis par les CFPA par rapport au système traditionnel polygame).

- chez les villageoises, absence assez généralisée d'activités féminines. Cette situation peut s'expliquer par 2 faits: d'une part les activités féminines représentent des thèmes spécifiques réservés aux femmes plutôt que des actions communautaires villageoises et intégrées. D'autre part les thèmes de formation sont demandés concrètement par les villages, c'est-à-dire par l'assemblée des hommes. (La situation d'instabilité socio-politique des dernières années a renforcé l'absence d'activités féminines).

Des contradictions entre une formation "théorique d'une activité féminine et les conditions pratiques de sa réalisation. En exemple, l'éducation sanitaire est donnée aux femmes et la responsabilité des pharmacies villageoises est confiée aux hommes selon un système social traditionnel. Autre exemple, la formation hygiène et santé s'adresse seulement aux femmes et ne fait pas partie de la formation entretien des puits réservée aux hommes.

Une progression discontinue des innovations.

- exemple en Suisse: en 1972 - 1973, la DDA avait une position assez avancée en intégrant l'animation féminine par une Africaine dans une expérience rurale. Or, les 2 évaluations qui ont suivi ont été confiées à des experts hommes en majorité ingénieurs, sans prévoir uné évaluatrice pour observer les conditions de réalisation de l'animation féminine (participation fortuite d'une évaluatrice en 1985). Dans la consultation des villageois, on n'a pas prévu méthodologiquement de consulter hommes et femmes en assemblées séparées, étant donné que les contraintes socio-culturelles d'une société de type patriarcal empéchent la femme de s'exprimer en réunion publique.

- exemple au Moyen-Chari, l'alphabétisation fonctionnelle en langue Sara a subi beaucoup de contretemps et de régression auprès des femmes.

Hybridation du traditionnel et du moderne. Introduction de changements sans rupture brutale comme intégration des techniques ou modèle de développement dans l'organisation sociale locale en fonction des systèmes de valeur villageois. En exemple, les démonstrations d'alimentation qui conseillent de donner des coufs aux enfants se heurtent à des tabous culturels. Le progrès apporté par une meilleure qualité nutritive des aliments doit être accoptable par leur système de valeurs.

Dynamique de transformation a long terme de la société locale. Outre les faits visibles du développement rural, cette région a enregistré de nombreuses mutations qui modifient en profondeur le milieu paysan (culture du coton et culture attelée, commercialisation, monétarisation, etc.). Dans la mêmé évolution, d'autres mutations encore peu visibles sont en cours qui transforment à long terme les fondements de cette société (scolarisation, certaine professionnalisation des formateurs, émergence de nouveaux pouvoirs et de nouveaux responsables villageois, consommation, individualisme). 
Formation en profondeur orientée sur la responsabilisation. La formation assurée par les CFPA a tenu compte de rythmes de développement differents: temps court des transformations techno-économiques (par exemple l'apprentissage des signes dans l'alphabétisation et des mécanismes élémentaires de calcul), temps long de l'évolution des mentalités qui assimilent les changements dans la durée d'une génération (par exemple le rôle de l'alphabétisation comme instrument de conscientisation sur les problèmes, de maîtrise de la commercialisation, et de responsabilisation pour assurer des activités villageoises autocentrées). Quant à leurs capacités de responsabilité, elles sont illustrées par la volonté déterminée et le dynamisme observé chez les villageois, qui ont subsisté malgré les événements socio-politiques des dernières années et les conditions d'instabilité et de destruction.

D'une manière globale, l'animation féminine n'a pas donné les résultats attendus. Les conditions d'échec peuvent s'expliquer par trois facteurs. Premièrement l'évolution historique a privilégié la formation des hommes créant un retard pour les femmes (animation féminine plus tardive et scolarisation encore rare des filles dans les écoles villageoises). Deuxièmement pèsent lourdement les contraintes sociales et culturelles d'une société patriarcale, bloquant souvent les femmes dans leurs initiatives et les soumettant à des projets pensés par des hommes. Troisièmement, les activités féminines proposées ne s'intègrent pas dans des actions communautaires villageoises, mais restent trop spécifiquement féminines.

Cependant, les femmes ne sont pas restées passives et spectatrices. En commercialisant leurs produits sur les marchés régionaux, elles se sont adaptées au développement et se sont intégrées à une économie marchande, suivant une évolution parallèle à celle des hommes mais avec un niveau de production beaucoup plus modeste que la commercialisation du coton ou des arachides. Pourtant cette adaptation spontanée, c'est-à-dire non programmée dans l'animation féminine, n'a pas modifié leur statut social.

Aujourd'hui, les femmes ont conscience de leur rôle dans la société villageoise. Elles demandent prioritairement une formation en agriculture et en alphabétisation fonctionnelle. La maîtrise de ces deux techniques est révélatrice d'un projet collectif rural. D'une part elles conçoivent leur rôle comme associé à des activités non féminines mais collectives pour hommes et femmes, basées sur la complémentarité et la polyvalence dans le travail et les rapports sociaux. D'autre part, elles visent une amélioration des conditions de vie de la famille et du village par une activité lucrative encore modeste, qui les fait sortir progressivement de l'autosubsistance vers une économie marchande avec ses nouveaux modèles de consommation.

Les monitrices chargées de l'animation féminine ont suivi elles aussi une certaine évolution dans leurs conceptions. Par leurs revendications salariales et professionnelles, elles représentent avec les moniteurs une nouvelle catégorie sociale. Cette classe sociale qui émerge actuellement est encore peu structurée mais déjà dissociée de la société villageoise et des problèmes locaux. 
Ces différentes tendances exprimées au féminin s'insèrent dans le contexte général d'évolution tchadienne. Depuis quelques décennies, cette région rurale du Moyen-Chari a enregistré de nombreuses mutations qui lui confèrent son visage actuel: l'introduction de la culture du coton et de la culture attelée, le développement du commerce, la monétarisation de l'économie, la pénétration de nouveaux modes et besoins de consommation, le changement des valeurs morales par la christianisation. Dans la même évolution, d'autres mutations sont en cours, encore peu visibles dans la vie quotidienne concrète, telles la scolarisation plus généralisée des enfants qui induit une certaine rupture entre générations, la naissance d'un nouveau pouvoir basé sur des connaissances techniques (écriture, calcul) et qui se confronte au pouvoir coutumier traditionnel, l'émergence de l'individualisme qui se substitue progressivement au sens communautaire. Et tous ces mouvements internes transforment en profondeur les rapports sociaux dans le village et dans la famille ainsi que les fondements de la culture rurale. C'est là que le rôle des femmes prend toutes ses dimensions, femmes partenaires d'un développement marchant sur deux jambes valides.

\section{Au Bangladesh, le Rural Advancement Committee (BRAC)}

Exposée très brièvement en trois niveaux de lecture parallèles à l'expérience tchadienne, l'initiative BRAC visait à améliorer la santé des enfants en milieu rural par le Oral Therapy Extension Programme (OTEP), développé ultérieurement en Child Survival Programme (CSP). Animation destinée aux femmes pour des occupations traditionnellement féminines, elle fut conçue comme une collaboration hommes-femmes: des équipes mixtes sensibilisaient la population villageoise, respectivement les leaders religieux et les autorités traditionnelles, ainsi que les mères de famille (7).

\section{Faits}

Création en 1980 par BRAC du Programme OTEP pour enseigner aux mères des régions rurales les techniques de rehydratation orale pour les enfants, ainsi que leurs conditions d'application.

1ère réorientation en 1983 visant une approche plus intégrée avec:

- élargissement de la formation santé axée sur les premiers soins et la nutrition

- approfondissement dans la formation dans la formation des monitrices, avec suivi des actions dans les villages

- action fourneaux (abandonnée par la suite)

2ème réorientation en 1986 avec lancement du programme plus global «survie de l'enfant":

- vaccinations, vitamine $K$, éducation nutritionnelle

- formation des matrones traditionnelles et contrôle prénatal 


\section{Evaluations en 1983 et 1986}

Animation a touché l'ensemble des familles dans les villages concernés (en 1985, près de 1400 animateurs - animatrices dans 7 régions, et plus de 6 millions de familles touchées en 1986).

Appui financier et technique de la DDA.

\section{Démarches}

L'action de formation s'est réalisée par ajustements successifs:

- à partir d'une approche unilatérale (réhydratation orale), tâtonnements et corrections ont conduit à une approche plus globale basée sur la santé, intégrant la sensibilisation aux soins de santé primaires et la participation des sagefemmes traditionnelles.

- méthode développée par BRAC de suivi incorporé à l'animation, sous forme de rapports journaliers, séances hebdomadaires et mensuelles des équipes d'animateurs-animatrices, a permis le contrôle et l'ajustement continuel des actions.

- 1 e et 2 e phases conduites de manière autonome, puis 3e phase en collaboration avec le gouvernement.

L'action de développement par la formation est liée à des innovations:

- $1 e$ innovation parmi les responsables de BRAC dès sa création de s'occuper de problèmes féminins et de concevoir des projets adressés aux femmes.

- innovation aussi dans l'approche utilisée, c'est-à-dire constituer des équipes mixtes de 7 femmes et 2 hommes se déplaçant de village en village, et former des animatrices spécifiques dans des domaines nouveaux (réhydratation orale, vaccination, etc.)

- innovation dans le système socio-culturel bangladeshi de promouvoir des comportements féminins qui sortent des normes: les animatrices travaillent et sortent de leur milieu familial et villageois pour le réaliser, se déplaçant à pied ou en rickshaw hors du contrôle social habituel.

- innovation encore dans le fait que des femmes extérieures aux villages s'adressent officiellement à des femmes.

L'innovation en tant qu'idée nouvelle est introduite de l'extérieur puis diffusée.

L'idée animation adressée aux femmes fut lancée par des Bangladeshi mêmes, mais des initiateurs intellectuels urbains, c'est-à-dire extérieurs au milieu rural de l'opération.

Les actions de développement sont des réponses aux besoins des collectivités. L'action répondait au départ à un besoin concret de lutter contre la mortalité infantile due à la diarrhée, et ses effets étaient concrets et visibles. La technique enseignée utilise des ingrédients disponibles dans chaque famille, et elle est résumée en un message simple: «seven points to remember". Programme conçu non comme projet féminin mais comme projet de santé, parce qu'il s'adresse à la population entière où les pères et mères de famille sont concernés par la survie de leurs enfants. 
Opération effectuée dans les familles, unités sociales traditionnelles de base.

La diffusion des innovations est assurée par des agents locaux.

Les animatrices bangladeshi sont originaires du milieu rural local, connaissant les besoins des populations et les contraintes sociales, et respectant le système des valeurs villageois.

\section{Effets}

Dans cette expérience lancée et réalisée par des Bangladeshi dans leur propre pays, la participation féminine est caractérisée par:

Une certaine conformité du rôle des femmes qui reste marginal en accord avec les valeurs locales, la sensibilisation touchant les comportements des femmes entre elles sans changer le système social global.

Un impact différencié. L'animation a touché femmes et hommes des villages bangladeshi de manière différenciée d'après leurs degrés de participation, selon 4 niveaux:

- les animatrices, scolarisées, formées aux techniques et connaissances nouvelles, dont le comportement «professionnel" est en rupture avec le milieu local traditionnel, sont de véritables agentes de développement et de changement.

- chez les villageoises, il y a conscientisation au 1er degré sur les problèmes de santé et la façon de les résoudre par des techniques nouvelles et appropriées. II y a changement dans le fait que les femmes en parlent ouvertement entre elles. Mais il n'y a pas encore de transformation en profondeur des rapports sociaux et du rôle de la femme dans la société.

- chez les responsables villageois, leaders religieux et autorités, il y a conscientisation par action directe des animateurs chargés de les sensibiliser sur la portée et les bénéfices de l'action.

- chez les villageois, on observe une conscientisation indirecte par les bienfaits apportés à la santé et au bien-être des enfants, qui permet de faire accepter l'animation des femmes.

Hybridation du traditionnel et du moderne. Les changements introduits sont acceptés parce que les «seuils de transformation" et les seuls « seuils de conformité" sont respectés. Hommes et femmes travaillent ensemble dans des équipes villageoises, mais leurs comportements à l'intérieur de ce groupe et dans les villages obéissent scrupuleusement aux règles sociales traditionnelles (par ex. les femmes ne se déplacent pas seules, portent un sari et couvrent leur tête en public, etc.)

La collaboration hommes-femmes est définie dans des tâches séparées: les animateurs établissent les contacts à la mosquée et au marché avec des hommes, leaders religieux et autorités, et les animatrices ont des contacts avec des femmes, sages-femmes et mères de famille. Les équipes introduisent des connaissances en provenance de la ville, mais elles se comportent en fonction des usages ruraux. Si les techniques enseignées sont modernes, par contre les méthodes d'animation ne sont pas utilisées contre ou aux dépens des chefs religieux et de la société locale, et elles ne créent pas de adésordre social». 
Dynamique de transformation à long terme de la société locale. Si les faits visibles de cette animation portent sur l'amélioration de la santé et la baisse de la mortalité infantile, il faut relever aussi une transformation en profondeur progressive sans rupture déséquilibrante qui touche au rôle de la femme. Par exemple, le comportement eprofessionnalisé" des animatrices hors de leur famille et de leur milieu villageois est accepté et considéré comme honorable, introduisant très lentement d'autres rapports au travail, à la famille et aux hommes.

Formation en profondeur orientée sur la responsabilisation. La formation assurée par BRAC a créé une sensibilisation généralisée à toute la population, qui s'adapte au rythme lent d'évolution des mentalités et qui donnera des résultats en profondeur dans la génération suivante, filles des première mères sensibilisées. Elément vital du projet, c'est la responsabilisation des acteurs sociaux: animateurs-animatrices devant assurer la réussite des actions dans les villages et assumer les difficultés, villageoises maitrisant mieux la santé de leurs enfants.

A partir d'une telle expérience, des conditions déterminantes de réussite se mettent en évidence. Dans cette initiative destinée aux femmes, on a évité le piège de marginaliser une «animation féminine» en centrant l'action sur la santé, domaine intégrant les besoins familiaux, le bien-être des enfants, et la satisfaction des parents. Basée sur la complémentarité des rôles et des fonctions entre hommes et femmes, elle s'est insérée dans une société rurale aux déterminismes sexuels très marqués. Les animatrices, comme agentes de changement, furent acceptées dans ce nouveau statut social, favorisant un ajustement progressif des comportements et une sensibilisation en profondeur sur le rôle des femmes. Contrairement à l'initiative tchadienne, cette action en cours est née au Bangladesh et elle constitue une expérience de développement endogène, pensé et réalisé localement.

\section{Un bilan double, lcl et là-bas}

Deux expériences, deux continents, deux scènes culturelles différentes au Tchad et au Bangladesh, avec des femmes «donneuses et receveuses de développement . Un bilan intéressant et convaincant. Un bilan officiel et officieux, c'est-à-dire lisible dans des rapports techniques et lisibles dans la société locale.

Loin d'un discours moralisateur j'aimerais relever six éléments du processus de développement, tels qu'ils ressortent de l'expérience conjointes des opérateurs et opératrices de la DDA, des promoteurs et promotrices ainsi que des populations villageoises du Tchad et du Bangladesh. Ils sont essentiels. Parce que l'enjeu primordial n'est pas de promouvoir un développement féminin, mais un développement socialement viable.

Autonomie individualiste contre intégration communautaire. Entre pays industrialisés et pays en développement, besoins fondamentaux et projets sociaux 
diffèrent fondamentalement. Ils déterminent des conditions de participation féminine et des motivations spécifiques. Dans les pays industrialisés adonneurs en développement», qui visent à accroître leur niveau de revenu et de consommation, la femme revendique ses droits à l'autonomie au sein d'une société où l'individualisme prédomine dans le système des valeurs. Dans les pays «receveurs du développement» qui luttent pour leur existence et leur survie contre la famine, la maladie, la pauvreté, les femmes cherchent à s'intégrer aux activités économiques et sociales pour le bénéfice communautaire, dans la famille et dans le village.

Des innovations successives, de l'extérieur vers l'intérieur. Toute démarche de développement passe par des phases successives d'innovations, introduisant et diffusant des idées et des conceptions nouvelles. Tâtonnements, essais, résistances, rejets, acceptations, corrections marquent leur parcours et traduisent leur expérimentation auprès des différents types d'acteurs sociaux (8). Parmi ceux-ci, nous distinguons principalement:

- les «innovateurs» ou ceux qui introduisent l'innovation. N'appartenant pas ou plus au milieu local, ils sont en contact avec des contextes culturels diversifiés, carrefours d'idées et d'expérimentations (par exemple, Européens au Tchad et intellectuels urbains au Bangladesh).

- les «formateurs" ou ceux qui sont formés aux idées nouvelles pour les diffuser dans la collectivité. Appartenant au milieu local et intégrés socialement, ils jouent le rôle moteur d'enclanchement du processus.

- les «premiers receveurs ou adoptants" qui vont assurer la diffusion des innovations. Jouant le rôle de relais, ils apparaissent comme des modèles à suivre pour les autres membres de la collectivité, et des interlocuteurs-interlocutrices privilégiés pour les formateurs (par exemple délégués au Tchad).

- la «population" dans son ensemble, plus ou moins avancée ou retardataire, c'est-à-dire les villageois et villageoises qui acceptent et maîtrisent l'innovation dans un délai plus ou moins long en fonction des réseaux d'information et de leur attachement aux valeurs locales. Pour chaque catégorie d'acteurs-actrices receveurs, lidée nouvelle perçue d'abord comme une anomalie dans les habitudes culturelles se normalise progressivement à mesure qu'elle est acceptée. Et la participation de la femme au développement passe obligatoirement par des innovations.

Dynamique de transformation aux rythmes différents. Ces phases successives d'innovations introduisent des rythmes différenciés dans la dynamique de transformation. Il existe un premier décalage entre les interventions techno-économiques qui se mettent en place rapidement dans un temps court, alors que le changement des mentalités progresse lentement et demande un temps long d'adaptation culturelle et sociale. Le deuxième décalage résulte de la diffusion des innovations entre les différents types d'acteurs pour qu'il y ait conscientisation en profondeur. Les expériences réalisées démontrent des temps relativement courts pour les formateurs (3 à 6 ans), des temps moyens d'une décennie pour les premiers receveurs-adoptants, et le temps long d'une génération pour la population (ce temps 
n'est pas encore échu pour les villageoises du Tchad et du Bangladesh). La transmission de l'innovation à la génération suivante est l'étape ultime et décisive qui marque l'intégration de la transformation.

Des lieux de créativité sociale. Les villageois et villageoises, derniers adoptants de l'innovation, ne sont pas simplement des rouages qui fonctionnent selon les impulsions données par des acteurs privilégiés. La manière dont ils interprètent les innovations en les corrigeant ou en les contournant représentent des réponses sociales. Au contraire d'obstacles à surmonter tels que les considèrent trop souvent les «développeurs", ces réponses correspondent à de nouvelles inventions culturelles. Créativité enracinée dans le système culturel local sans contredire ni ses valeurs ni ses pouvoirs de décision, elle contribue à la continuité sociale faite de participation complémentaire hommes-femmes.

Emergence de forces sociales et contre-pouvoirs nouveaux. Dans la progression des changements observés dans les pays en développement, il y a décalage entre les urbains et les ruraux, entre les intellectuels et les ouvriers, entre les entrepreneurs et les paysans. Ainsi, parallèlement à une continuité socioculturelle en profondeur, de nouvelles forces sociales émergent progressivement, telles les moniteurs-monitrices ou les responsables villageois d'activités communautaires. Selon les conditions, ils seront acceptés comme nouveaux leaders ou au contraire craints comme contre-pouvoirs plus techniques et modernes créant des tensions avec les autorités coutumières traditionnelles. Ces courants actifs et réactifs traversent continuellement les collectivités, et les femmes peuvent y trouver des rôles et des voies privilégiés dans la mise en marche d'un changement.

Identité des femmes. L'identité des femmes dans le développement n'existe pas comme donnée de départ. L'identité - image qu'on a de soi - ne se révèle que par rapport à l'image qu'on a des autres considérés comme différents. La participation de la femme peut être cette occasion de coupure et de distanciation qui lui permet de prendre conscience de sa position et de son rôle potentiel dans le développement. Image neuve qui la figure non comme un rouage subordonné, mais comme un élément-clé de la mécanique motrice. Des femmes l'ont déjà prouvé en participant au développement de leur village ou à l'indépendance de leur pays.

\section{Vers une "féminitude» impliquant la complémentarlté hommes-femmes}

Dans les pays industrialisés, les efforts s'intensifient pour multiplier les aides au développement et la réflexion critique s'approfondit pour réorienter les finalités du développement.

Comment continuer la route sur la voie du développement au féminin? II y a plusieurs réponses nuancées: ne pas isoler la femme et en faire une catégorie marginalisée, ne pas lui créer de nouvelles dépendances, mais renforcer sa coopération avec les autres groupes sociaux et la faire participer aux projets collectifs. 
C'est aussi aider les sociétés à reconnaître le rôle des femmes, à leur permettre de s'exprimer et à leur donner les moyens d'agir. Pour ceci des animatrices sont nécessaires certes, mais aussi des leaders masculins. Des monitrices peuvent œuvrer dans les difficultés quotidiennes mais les responsables locaux doivent aussi promouvoir et légitimer les réalisations.

"L'égalité» hommes-femmes revendiquée entre les sexes n'a pas la même image en Europe ou en Amérique latine. Qu'elles soient Maliennes, Boliviennes ou Indonésiennes, les femmes vivent autrement leurs rapports aux hommes, aux enfants, à la famille, au village, à la société entière. Chaque société fixe ses normes dans les responsabilités et les droits, dans la complémentarité sociale et l'équilibration des rôles, dans ses définitions spécifiques du masculin et du féminin.

La civilisation occidentale a conquis certains droits fondamentaux. Successivement, pendant plusieurs décennies elle a acquis le droit à la santé, le droit à la nourriture, le droit au travail, le droit à l'éducation, le droit aux loisirs, le droit à la parole féminine. Les pays en développement sont en train d'accéder à tous ces droits simultanément, situant les «droits féminins» au cœur du développement mais non comme une revendication d'autonomie.

D'une manière générale, les valeurs féminines s'incarnent progressivement dans la société et deviennent opérantes. Mais le cheminement par tâtonnement progresse dans des zones d'ombre, dans des phases de flou et d'incertitude qu'on veut trop souvent corriger définitivement par des programmes précis et contraignants. Or, ceux-ci œuvrent à contresens parce que les besoins de la société et les besoins des femmes se négocient en continu.

A l'aube du 3 e millénaire, Edgar Morin parle de la création d'un nouvel «archétype culturel fondé sur l'association complémentaire des valeurs: masculinféminin, occident-orient, nord-sud, rationalité-poésie... (9). Le féminin est en marche. Vers une nouvelle société du 21 e siècle, nouvel âge pour les femmes et les pays en développement?

\section{Références}

1. ONU, "Déclaration sur l'élimination de toutes les formes de discrimination à l'égard des femmesn, Commission de la Condition de la femme, 1967.

2. Cf. iзM et CETIM, «Femmes: une décennie pour s'entendre", dossier i3M $n^{\circ} 16$, Publiḉtim $n^{\circ}$ 15, Lausanne, Genève, 1986.

3. Le bulletin de la DDA «Antennem, $n^{\circ} 4$, décembre 1983, présente les lignes directrices rédigées par le Service sectoriel Développement social. II peut être obtenu auprès du Service de l'Information de la DDA.

4. Cf. E+D, «La situation de la femme dans le Tiers-Mondem, $n^{\circ} 7$, DDA, 1980, et en particulier l'article de Immita Cornaz aPrendre les femmes par la main ou leur donner le droit de parole?"

5. Voir le «Rapport sur la politique de Coopération au développement 1976-1985», Berne, 2 mars 1987.

6. Voir le «Rapport de situation des Centres de formation professionnelle agricole (CFPA) du Moyen Chari", Sahr, 14 janvier 1985; le "Compte-rendu du séminaire sur les CFPA 
au Tchad, 30.09. au 03.10.1985 à Moundoun, N'Djamena, par Perrier Régis; le «Rapport de la mission d'évaluation des CFPA du Moyen-Chari au Tchad ", DDA, 1985, par Lieberherr-Gardiol Françoise et Ryser Daniel; complément d'information par Immita Cornaz, Service sectoriel Développement social, DDA.

7. Voir rapports "Evaluation of the oral therapy extension programme (OTEP) of the BRAC", phase 1 (1980-83) par Bhatia Shushum, Cash Richard et Cornaz Immita, phase 2 (1983-86) par Cornaz Immita et Pyle David F.; compléments d'informations par Immita Cornaz et Annick Tonti, DDA.

8. Voir parmi les recherches sur les acteurs de l'innovation Bodiguel M., « Les paysans face au progrès", Paris, Presse Fond. Nat. Sc. politique, 1975, et Bassand M., Hainard F., Pedrazzini $Y$. et Perrinjaquet R., «Innovation et changement social», Lausanne, Presses Polyt. romandes, 1985.

9. Morin Edgar, «L'esprit du temps, 2, Nécrose», Paris, Grasset, 1975, page 263. 